\title{
INTERNACIONALIZACIÓN DE LA EDUCACIÓN SUPERIOR EN LATINOAMÉRICA, ¿CÓMO APORTA EN LA SOSTENIBILIDAD?
}

\section{INTERNATIONALIZATION OF HIGHER EDUCATION IN LATIN AMERICA, HOW DOES IT CONTRIBUTE TO SUSTAINABILITY?}

\author{
Iván Leonardo Medina-Alvarado 1 \\ Fundación Universitaria del Área Andina y Universidad Santo Tomás \\ Doctor (c) en Educación y MBA \\ ivan.medina.alvarado@gmail.com
}

\begin{abstract}
Resumen: Los procesos de internacionalización de la educación superior en Latinoamérica, al igual que el mundo entero durante el siglo XXI, han aumentado significativamente su protagonismo en la agenda educativa de nivel superior, siendo una práctica que se presenta desde múltiples opciones y estrategias, todas estas conducentes a fortalecer los paradigmas de la educación globalizada y de contexto para con el mundo moderno; ahora bien, cada rincón del mundo ha adoptado múltiples opciones para favorecer estas prácticas de internacionalización más aun, cuando la pandemia generada por el COVID-19 posibilitó el imaginar la educación de una forma muy diferente, lo cual indica, que no se puede pretender adoptar un único modelo que permita alcanzar los indicadores y metas que en cada centro de educación superior se presentan en términos de internacionalizar la educación y los procesos educativos; así las cosas, este documento presenta un análisis sobre estas prácticas que se llevan en el entorno latino. De igual forma, se busca comprender cuál es el aporte que la internacionalización ha generado a favor de la sostenibilidad, entendida esta última, desde la perspectiva social, económica y financiera; de manera que se buscan identificar los aspectos relevantes que denotan una contribución a favor de un mundo más sostenible, que se ha visto obligado a cambiar su forma de actuar a raíz de la pandemia que trajo el siglo XXI.
\end{abstract}

Palabras clave: internacionalización educativa, COVID-19 y educación superior, sostenibilidad y educación, educación superior sostenible, multiculturalidad universitaria.

Resumo: Os processos de internacionalização da educação superior na América Latina, como em todo o mundo durante o século XXI, têm aumentado significativamente seu destaque na agenda educacional de nível superior, sendo uma prática que se apresenta a partir de múltiplas opções e estratégias, todas. propício ao fortalecimento dos paradigmas da educação globalizada e do contexto para o mundo moderno; Porém, cada canto do mundo adoto u múltiplas opções para favorecer ainda mais essas práticas de internacionalização, quando a pandemia gerada pela COVID-19 possibilitou imaginar a educação de uma forma muito

\footnotetext{
${ }^{1}$ Profesor universitario en pregrado para la Fundación Universitaria del Área Andina y Universidad Santo Tomás, Bogotá D.C. Colombia.
}

Fecha recepción: 13 de enero de 2021

Fecha aceptación: 8 de septiembre de 2021 
diferente, o que indica que não se pode pretender adotar uma única modelo que permite atingir os indicadores e objetivos que se apresentam em cada centro de ensino superior em termos de internacionalização da educação e dos processos educativos; Assim, este documento apresenta uma análise dessas práticas que são realizadas no ambiente latino. Do mesmo modo, procura compreender qual o contributo que a internacionalização tem gerado a favor da sustentabilidade, compreendendo esta, do ponto de vista social, económico e financeiro; De forma a identificar os aspectos relevantes que denotam uma contribuição a favor de um mundo mais sustentável, que se viu obrigado a mudar a sua forma de agir em consequência da pandemia que o século XXI trouxe.

Palavras-chave: internacionalização educacional, COVID-19 e educação superior, sustentabilidade e educação, educação superior sustentável, multiculturalismo universitário.

Abstract: The internationalization processes of higher education in Latin America, like the whole world during the XXI century, have significantly increased their prominence in the higherlevel educational agenda, being a practice that is presented from multiple options and strategies, all of these leading to strengthen the paradigms of globalized education and context for the modern world; However, every corner of the world has adopted multiple options to favor these internationalization practices even more, when the pandemic generated by COVID19 made it possible to imagine education in a very different way, which indicates that it cannot be intended to adopt a single model that allows to achieve the indicators and goals that are presented in each higher education center in terms of internationalizing education and educational processes; Thus, this document presents an analysis of these practices that are carried out in the Latin environment. Similarly, it seeks to understand what is the contribution that internationalization has generated in favor of sustainability, understanding the latter, from the social, economic and financial perspective; In such a way that they seek to identify the relevant aspects that denote a contribution in favor of a more sustainable world, which has been forced to change its way of acting as a result of the pandemic that the 21st century brought.

Keywords: educational internationalization, COVID-19 and higher education, sustainability and education, sustainable higher education, university multiculturalism.

\section{INTRODUCCIÓN}

El paradigma de la educación superior representa una constante hazaña al interior de los sistemas educativos, situación que en el caso de Latinoamérica se caracteriza por guardar una cercana relación al interior de gran parte de sus países, destacando aspectos de tipo: social, cultural, económico entre otros; es por esto que para López-Segrera (2006), resulta necesario comprender como desde uno de los elementos a considerar de la educación superior, el cual en esta oportunidad es la internacionalización, se aporta en todo el conjunto de acciones que denotan la sostenibilidad no solamente desde la perspectiva de cada centro de educación, sino entendida esta, como un conjunto de compromisos que adquiere una comunidad ya sea educativa de cualquier tipo, a favor de prácticas responsables para con el medio ambiente, los 
recursos naturales y la sociedad; más aún cuando situaciones detonantes como lo es la pandemia generada por el COVID-19, invitan a replantear lo que históricamente se ha venido proponiendo en términos de educación superior

Con el paso del tiempo la internacionalización y las tareas que se generan favor de internacionalizar la vida universitaria, se han visto enriquecidas por cientos de prácticas y escenarios en los que se comparten experiencias a favor de una propuesta educativa internacional; ahora bien, este documento analiza desde una revisión bibliográfica que enmarca la realidad y el conjunto de situaciones que se viven en gran parte de Latinoamérica, las acciones más destacadas en términos de internacionalización; de igual forma esta revisión procura identificar como este ejercicio de internacionalizar la educación superior, ha contribuido a fortalecer prácticas de sostenibilidad, ya sea en: el currículo, la oferta educativa, prácticas empresariales, investigación, proyección y extensión universitaria.

Elementos como la responsabilidad social universitaria -RSU- según Chacón, Montbrun, y Rastelli (2009), hacen parte del quehacer diario en una institución de educación superior y se ratifican como el epicentro de múltiples caminos a favor de la sociedad, y a la vez proporcionan escenarios para fortalecer incrementar el relacionamiento con las comunidades menos favorecidos; estas prácticas si bien no son nuevas, si han cobrado mucho protagonismo en la sociedad actual, donde la información es más accesible para el público objetivo, y le proporciona a las comunidades elementos de valor al momento de medir el valor de una imagen institucional, es así como, en Latinoamérica la RSU año tras año ha logrado impactar las organizaciones educativas en términos de número de estudiantes, relacionamiento con el sector externo, cualificación docente y hasta las formas de gobierno educativo en términos del llamado, valor compartido.

Paradójicamente en América Latina, muchas universidades han identificado la internacionalización como un elemento complementario o adicional de los procesos de enseñanza que se gestan en las aulas, de allí, que desde la mirada de Monarca Prieto (2018), gran parte de las comunidades educativas den mayor prioridad que otras, en términos de fortalecer el portafolio de tareas académicas que se desarrollen fuera de las fronteras de un país, por otro lado, la sostenibilidad en algunas instituciones de educación superior se ha ratificado como un elemento del proceso de administrativo y organizacional, donde se pretende hacer alusión a este concepto desde la misma mirada de las empresas, quienes adoptan la sostenibilidad de los parámetros de la Responsabilidad Social Empresarial RSE y la Responsabilidad Social Corporativa RSC, esto ha denotado en grandes conflictos para con la forma en que la educación superior es percibida por la sociedad, ejemplo de esto se ha visibilizado por la pandemia generada por el COVID-19, llegado a cuestionar la importancia de la educación superior, gracias a inadecuados manejos del concepto de sostenibilidad en la organización educativa, la cual no puede ser vista desde la misma mirada de una empresa que busca un lucro o una utilidad, sino que se debe traducir en el impacto social que esta genera a la sociedad. 


\section{PROCESOS DE INTERNACIONALIZACIÓN EN LAS UNIVERSIDADES LATINAS}

Cuando se hace referencia a la internacionalización de la educación superior, sin igual a dudas se debe aludir a las acciones que desde la dirección educativa en una universidad se han propuesto para favorecer la vida universitaria tal como lo presenta Gijón-Puerta y Crisol-Moya (2012), todo esto orientado desde una identidad estratégica que se articula con los lineamientos establecidos en un proyecto educativo institucional PEI, esta planeación educativa requiere una detallada organización y articulación en términos de las tres funciones sustantivas de la educación superior como lo son: la docencia, la investigación y la proyección social o también llamada extensión universitaria; de manera que la internacionalización se presenta como un elemento transversal de la propuesta organizacional de cada universidad.

Cuando se analizan las acciones que se ejecutan en algunos centros educativos en Latinoamérica, se evidencian ciertas tareas que se generan a favor de una educación superior internacionalizada, aun así, no en todos los casos se existe una marca relación entre la planeación estratégica y la orientación de estas tareas, de manera que la ardua labor que deben asumir los directores educativos junto con sus equipos de trabajo, debe estar orientada no solamente a cumplir con una serie de indicadores, llevando a comprender la internacionalización como un elemento transversal que está presente en todo el ejercicio educativo, y que no se limita únicamente a las actividades del aula de clase.

Para comprender los aportes más significativos que trae consigo la internacionalización en las universidades latinoamericanas, a continuación, se presentan tres importantes elementos a considerar, los cuales son el común denominador al momento de consolidar una cultura educativa internacional.

\section{Intercambio cultural}

La educación superior se destaca por su importante contribución para con las causas sociales, de allí que la sociología de la educación ratifique el importante rol para con el desarrollo social y progreso de las regiones, esta realidad en el contexto latinoamericano tiende a ser mucho más protagónico que en el resto del mundo, precisamente porque la riqueza cultural en américa latina es alta y adicional la heterogeneidad cultural posibilita el interacción constante entre diversas cultural, grupos sociales e instituciones que orientan su accionar a favor de conservar la riqueza social, ahora bien, para Medina-Alvarado (2019) estas realidades culturales no siempre han permitido que la internacionalización educativa en el nivel universitario tenga una contribución positiva, toda vez que, en Latinoamérica países como Perú, México, Ecuador, Colombia, Bolivia, Chile y Argentina, cuentan con grupo sociales autóctonos, a quienes la internacionalización educativa en algunos casos ha traído consigo que se vulnere el valor ancestral y cultural.

Cuando se hace referencia a intercambio cultural desde la perceptiva educativa, AriasVelandia (2018), comparte que es necesario dejar claro, que se alude a un proceso de trasmisión de conocimientos ancestrales, de identidad social, entre otros; de manera que como indica Jones y Brown (2016), no se puede malinterpretar como un proceso que pretende traer una cultura o civilizar globalmente a las regiones, y precisamente la cultura globalizada en muchos aspectos como lo es la educación, ha generado como consecuencia una pérdida de identidad en muchas 
regiones latinas, siendo una situación que además de estar presente en el quehacer de la sociedad, hace parte del valor cultural inmaterial de las regiones.

Es relevante mencionar que en Latinoamérica si bien hay una alta concentración de comunidades indígenas y autóctonas, para quienes los procesos de internacionalización deben servir como un proceso de divulgación cultural, de comunicación de saberes únicos que garanticen su valor cultural, aun así, no todos los pueblos o regiones latinas se reconocen como comunidades autóctonas, y es allí, donde Brunner (2010) propone que la educación superior esta Ilamada a comprender que la identidad social se debe conservar y cultivar desde todos los niveles de la educación, entendida esta está como la articulación entre los diferentes niveles educativos, que comprenden la educación preescolar, primaria, media y superior, en la cual las comunidades educativas deben generar una planeación educativa, en la cual la coherencia entre niveles educativos y realidades sociales deben dar cuenta de los programas, planes y proyectos en términos de educación.

Para comprender la dinámica del intercambio cultural en América Latina desde la educación superior, Medina (2019) destaca importantes escenarios e iniciativas que en algunos gobiernos ha representado una conservación cultural, desde la cual el mundo entero a conocido prácticas únicas que dan cuenta de la riqueza cultural en el ecosistema latino, es por esto que estas contribuciones han hecho que se promueven aspectos como:

- Conocimientos en agricultura, ganadería, pesca y en general labores cotidianas de los pueblos latinos.

- Estructural sociales y de gobierno, que durante miles de años han estado presentes.

- Sistemas educativos y de conocimiento ancestral, que denotan una transmisión de saberes entre generaciones.

En algunos centros educativos de nivel superior en Latinoamérica, el intercambio cultural desde acciones de internacionalización educativa, ha permitido que sean positivas las iniciativas tareas que se han promovido desde el conocimiento conjunto y divulgación de la identidad cultural, lo cual ha logrado fortalecer los vínculos sociales al interior de pueblos indígenas, negritudes y todo el conjunto de comunidades con alta representación cultural, que día a día han hecho que las prácticas de internacionalización sean comprendidas no como ventanas al mundo para adoptar modelos externos, sino como rutas de comunicación colectiva de conocimiento e intercambio de saberes.

\section{Multilingüismo y nuevas ciudadanías}

Los idiomas y todo lo que gira entorno a comprender como se generan los procesos de comunicación desde el lenguaje en diversas partes del mundo, es sin igual a dudas uno de los elementos más importantes que se presentan al momento de comprender el impacto positivo que trae la internacionalización en la vida universitaria, no solo para el estudiante, sino para todos y cada uno de los miembros de una misma comunidad educativa, es por esto que se concibe un vínculo muy fuerte para con las nuevas ciudadanías, toda vez que estas nuevas ciudadanías 
representan el comportamiento de las personas que están haciendo parte de un grupo social, según su edad, contexto social, interés cultural, entre otros.

En la educación superior Latinoamericana, el multilingüismo para Blanco, Alba-Ferré y Asensio (2016), se ha consolidado como una tarea por la cual se deben aumentar drásticamente los esfuerzos, toda vez que, las estadísticas en términos de bilingüismo son bastante precarias con relación a otros lugares del mundo como lo son Europa, Asía y Norteamérica; ahora bien, según Aznar-Minguet, UII, Piñero y Martínez-Agut (2014), esta realidad está directamente relacionada con el desempeño que tienen muchos de los sistemas educativos en términos de consolidación de una cultura transversal hacia el fortalecimiento del bilingüismo y multilingüismo al interior de los procesos educativos, siendo nuevamente ratificada la falta de articulación entre los diferentes niveles educativos, como el detonante de esta situación.

Los procesos de internacionalización de cara al fortalecimiento del bilingüismo como valor agregado de los procesos de educación en la vida universitaria, requiere grandes inversiones y la presentación de alternativas que conjunten la pedagogía y la andragogía en las tareas de enseñanza y difusión de un segundo o tercer idioma como competencia profesional tal como lo menciona Pedroza-Flores (2005); frente a esta alternativa en Latinoamérica algunas universidades sobresalen al contar con laboratorios de idiomas, movilidad académica estudiantil y de docentes, actividades de investigación conjunta y un amplio portafolio de actividades de la vida universitaria que van más allá de la obtención de un título académico, sino que proveen de escenarios que desarrollan competencias para la vida y que aportan en gran manera en el éxito profesional.

En Latinoamérica, es necesario el aumento de incentivos al interior de cada centro de educación superior, de manera que estos incentivos se ven reflejados en cientos de acciones que den cuenta de la intención por incluir en la formación integral de los estudiantes, las competencias en el manejo de nuevos idiomas, y proporcionar un trabajo mancomunado entre los propósitos mismos del aprendizaje en términos de currículo y las competencias requeridas para comprender y superar las expectativas del mercado globalizado que propone esta era posmoderna.

\section{Internacionalización del currículo}

En un centro de educación superior la labor de gestión curricular y todo lo que implica una articulación detallada en cada programa académico que compone la oferta educativa ya sea de nivel de licenciatura y posgrado, ratifican la importancia de proveer e incluir en la ruta académica la internacionalización como un valor agregado, así las cosas, tal como lo menciona Castro (2018), internacionalizar el currículo de un programa académico de nivel universitario, es el resultado de un proceso de planeación minucioso y muy detallado que da cuenta de las materialización futura de una premisa que antecede la filosofía misma de una institución de educación superior, en escenarios como en Latinoamérica este proceso debe está orientado por la pertinencia de la oferta educativa y las realidades sociales que se viven en los diferentes grupos poblaciones, sin desconocer el aporte para con el desarrollo local, regional, nacional e internacional de cada individuo. 
Tradicionalmente según Ricardo-Barreto y Iriarte-Díazgranados (2017), las instituciones educativas desde comités por área y facultades, organizan tanto sus planes de trabajo como el conjunto de labores que se deben ejecutar periódicamente frente a la actualización y revisión del cumplimiento en los estadales curriculares según corresponda, siendo una labor que se desarrolla continuamente y debe gozar de altar participación, no solo de docentes y líderes de área, sino de estudiantes, administrativos, configurándose así como un punto de partida para encontrar oportunidades de mejora en el quehacer educativo; para gran parte de las universidades y centros de educación superior en Latinoamérica esta situación no es ajena y periódicamente demuestran esta importante labor en diferentes informes y herramientas de gestión, lo paradójico de este ejercicio, es que precisamente muchos de los centros educativos no incluyen la internacionalización y el intercambio de experiencias con otras comunidades educativas en esta labor o es considerada esta labor como una tarea de una dependencia ajena al ejercicio curricular.

Cuando se revisan los indicadores que presenta algunos sistemas educativos en América Latina, tomando como ejemplo en: Chile, Argentina, México, Costa Rica, Perú, Colombia, entre otros; se identifica que la internacionalización es vista como una labor de complemento o como el conjunto de acciones que se orientan a favorecer una experiencia internacional para la población educativa, situación que conlleva a identificar la gran diferencia para con el comportamiento Europeo y Norteamericano, siendo estos últimos destacados porque incluyen desde el componente del currículo la internacionalización no como un complemento, sino como un eje central para el cumplimiento de los resultados de aprendizaje y alcance de los objetivos de cada asignatura o espacio académico que compone un plan de estudios, así las cosas, para Salazar (2012), es de suma importancia considerar la internacionalización del currículo no solamente como una acción más del ejercicio educativo, sino como una herramienta que orienta la finalidad misma un programa académico.

Existen diversos puntos de vista entorno a la internacionalización currículo, salvo que desde los postulados de Meza-Aliaga (2020), el común denominador en ese paradigma, se ratifica al comprender que los ejercicios de internacionalización hacen parte de los cambios globales por los cuales el mundo entero está pasando, encontrando como punto de partida, los cambios en los procesos de comunicación, interrelación social, mercados globales, movimientos políticos, entre otros; dicho en otras palabras, la internacionalización del currículo no se genera como una respuesta a los cambios actuales, sino que obedece a la evolución misma del comportamiento humano y la sociedad actual.

\section{SOSTENIBILIDAD EN LA EDUCACIÓN SUPERIOR}

Entender la sostenibilidad al interior de una organización educativa se configura en un reto que solo se puede abordar desde la mirada de la Responsabilidad Social Universitaria RSU, y es que para Parrado-Castañeda y Trujillo-Quintero (2015), precisamente al hacer referencia a la sostenibilidad en la educación superior, se abre un amplio abanico de posibilidades que se traducen en tareas que se enfocan a contribuir socialmente en un entorno especifico, de allí, que para algunas universidad latinas el proponer prácticas de sostenibilidad es visto únicamente desde la mirada administrativa y desconoce el componente misma de cada institución de 
educación superior, por lo tanto, según Brown y Pickford (2016), los centros de educación superior deben proponer una detallada organización en sus actividades, tanto administrativas como educativas, en las que la sostenibilidad se integre al ADN de la organización y de forma conjunta se consolide como un valor agregado.

En Latinoamérica al igual que gran parte del mundo entero la sostenibilidad se ha adoptado y aprendido en cierta medida, desde el escenario empresarial donde desde la revolución industrial se ha propuesto la importancia de proponer acciones, planes, programas y proyectos que prologuen la vida misma de una empresa, y es así como para Cuenca (2013), la sostenibilidad desde sus diferentes reportes e indicadores, es visto como un pilar elemental de la trasformación interna y externa desde una labor económica, social política y de todo tipo, toda vez que la sostenibilidad implica la optimización de los recursos tanto presentes como futuros, llevando a proponer escenarios futuros como estancias de participación global, y es precisamente desde la premisa de comprender que la sostenibilidad no se puede abordar como un capricho local o gubernamental, sino como un necesidad global, es que en la educación superior la internacionalización se configura un aliado estratégico para favorecer la organización educativa y la comunidad en la cual esta hace presencia.

Año a año las instituciones educativas proponen sus cronogramas de metas y establecen los objetivos que se deben proponer en términos organizacionales, los cuales son trasladados a los colaboradores que hacen parte de cada institución educativa, en cierta medida esta práctica no es ajena al manejo organizacional que se lleva a cabo en una empresa que atiende a prácticas de RSE y RSC, ahora bien, según Hérvas-Gómez, Vásquez-Cano, Fernández-Batanero y LópezMeneses (2019), al comprender que una institución educativa no es una empresa que genera una utilidad a sus socios, hace que las metas y objetivos sean compartidos por la misma comunidad y esto implica no solo los colaboradores o trabajadores de la misma, sino a los integrantes que no tiene un vínculo laboral, tal es el caso de: los estudiantes, sus familias, los proveedores, el gobierno público, las otras instituciones educativas y en general son todo el conjunto de actores que se ven afectados directa o indirectamente, denominados stakeholders o grupos de interés.

Cuando una organización educativa comprende que sus grupos de interés no son ajenos a la realidad de la comunidad educativa, es cuando se genera el binomio para con la Responsabilidad Social Universitaria RSU, y esta a su vez da el protagonismo esperado a la sostenibilidad social, a la cual todas las organizaciones educativas están llamadas a fortalecer y priorizar desde su existencia misma, alienada con la planeación estratégica que se provee desde una sólida filosofía organizacional.

\section{Responsabilidad social universitaria}

Varios autores en el siglo XXI han abordado la Responsabilidad Social Universitaria, -en adelante RSU- y han propuesto un sin número de acciones que den cuenta de la incidencia de esta en la existencia de las organizaciones educativas, algunos han llegado a proponer la marcada necesidad de contar con políticas de RSU como mecanismo de interacción para con sus stakeholders; lo cierto es que, para Gacel-Ávila (2003) en el contexto latinoamericano las universidades y centros de educación superior aún no han comprendido en su totalidad lo que 
representa la RSU y es precisamente desde esta mirada que se propone comprender la incidencia de este movimiento a favor de la sostenibilidad, ratificando el rol que tiene la educación y los procesos educativos para con el desarrollo social.

La RSU se presenta como una declaratoria institucional para con el entorno y las comunidades en las cuales las organizaciones educativas tienen incidencia, de allí que para Meneses y Perea (2012), esto hace que estas prácticas no se configuren como un modelo único o como un conjunto de parámetros previamente establecidos, así las cosas:

- Lo primero que se debe comprender al aludir a la RSU, es que esta declaratoria institucional es única $y$, por lo tanto, no puede ser adoptada o copiada de otra organización educativa, resultado incoherente pretender asumir los mismos compromisos de una organización para con otra comunidad educativa o entorno en el cual se genera una interacción.

- La segunda premisa a considerar es que la RSU se debe estructurar desde la identificación y gestión de relaciones con sus stakeholders, así las cosas, las instituciones de educación superior deben hacer una detallada revisión de sus grupos de interés, delimitando prioridades, definiendo impacto directos e indirectos para más adelante proponer iniciativas que guarden cercanía para con el tipo de stakeholders y el impacto que se le genera.

- La tercera consideración, se orienta a complementar e integrar el panorama ya identificado para con la planeación estratégica de la organización y esto requiere que cada universidad, integre sus procesos académicos para con las necesida des e impactos generados a los grupos de interés, de manera que no solo es identificar los grupos de interés, sino que se proponen alternativas que guardan pertinencia y cercanía entre los grupos poblacionales, la oferta educativa y la labor misma de cada institución de educación.

- Como cuarto escenario, se deben integrar estos grupos de interés en las actividades con las cuales se pretende atender para dar cumplimiento a la declaratoria que cada institución de educación superior tiene, y es en este escenario, en el cual es de suma importancia recordar que al ser una declaratoria única con la que cuenta cada organización educativa, se pueden generar acciones conjuntas entre instituciones educativas de todo tipo y no solo organizaciones educativas, sino cualquier tipo de organización legal y que tenga de por medio un compromiso conjunto o cercano.

- El último escenario que se propone en términos de dar cumplimiento a la RSU en una institución de educación superior, se presenta la divulgación de las tareas, participaciones, impactos y resultados obtenidos desde un ejercicio mancomunado, por lo tanto, este escenario alude a la muy conocida, presentación de informes de sostenibilidad, entendidos estos informes, no como obligaciones o tareas adicionales de la labor ya realizada, sino como la consolidación de información relevante desde la cual se ratifica la declaratoria institucional en términos de sostenibilidad, así las cosas la RSU y la sostenibilidad van de la mano en el ejercicio educativo.

Si bien es cierto que la RSU y la sostenibilidad se consolidan como un binomio, para BarbaÁlvarez (2012), las instituciones educativas en Latinoamérica pareciese que las identificaran como 
actividades independientes, lo cual indica, que el grado de desconocimiento desde la administración educativa y la gestión de procesos educativos un es evidente, de allí que un vez más se confirma que la labor de una institución educativa no se puede comparar con una empresa y si propone acciones específicas para articular la organización educativa para con el compromiso social que toda universidad asume desde su existencia y su filosofía institucional.

\section{Valor compartido en la educación superior}

El concepto de valor compartido es otro elemento que se ha generado desde el siglo XX, cuando se procura abordar las necesidades sociales de un grupo poblacional, y es desde allí como las organizaciones educativas deben tener claridad que no se puede traducir este concepto con las mismas consideraciones de las empresas, para lo cual, para Chirinos-Araque y Pérez-Peralta (2016), es claro indicar que el valor compartido en una institución educativa es el resultado de la integración de los grupos de interés previamente identificados desde articulación de la RSU y la sostenibilidad, donde la generación de soluciones o alternativas de solución son colectivas y de tipo participativo, dicho en otras palabras, el valor compartido en una institución educativa, no se puede mal interpretar como el conjunto de tareas filantrópicas y altruistas que son vistas desde una mirada asistencialista, sino que deben ser vistas como el conjunto de tareas que con llevan a trasformar positivamente las regiones, de manera que sus habitantes y grupos poblacionales sean quienes protagonicen el cambio y no solo esperen ayudas humanitarias.

En el escenario latinoamericano, según Vallaeys (2018), el concepto de valor compartido desde la mirada de los centros de educación superior aun no es correctamente abordado tanto por las instituciones educativas, como por lo mismo grupos poblaciones a quienes se les ha identificado como grupos de interés, y una de las razones por las cuales esta situación está presente, obedece al desconocimiento que hay entre una verdadera práctica de RSU y lineamientos articulados para favorecer la sostenibilidad, entre ellos -y de forma errónea- la internacionalización de la educación superior, la cual no es vista como un punto céntrico que favorece y optimiza las acciones de sostenibilidad, limitando a las prácticas de internacionalización únicamente como un elemento complementario de la vida universitaria y desaprovechando la posibilidad de:

- Comprender nuevas formas de concienciar el cuidado por el medio ambiente.

- Consolidar una cultura ciudadana que de cuentas de compromisos claros a favor de la sostenibilidad en un futuro próximo.

- Incrementar la posibilidad de comprender nuevas culturas que han adoptado la sostenibilidad como un valor agregado de la existencia misma de la humanidad.

- Identificar prácticas exitosas de sostenibilidad que pueden ser replicadas o servir de orientación para transformar las regiones.

- Analizar las consecuencias del desconocimiento hacia conserva el medio ambiente y prevenir situaciones que no se puedan prevenir.

- Adaptar los recursos para prever soluciones a las necesidades actuales, a partir de la consolidación de una cultura de divulgación del conocimiento y experiencias sostenibles. 
Otro elemento por considerar desde el valor compartido, es la posibilidad de interactuar con todos los actores que hacen parte de una misma realidad o situación conflictiva, es así como la interacción constante y la comunicación efectiva deben estar presentes en todo momento cuando se hace referencia a proponer soluciones colectivas, y no imponer posturas o ideologías contrarias a las y establecidas por algunas comunidades, toda vez que, esto trae consigo consecuencias adversas a las esperadas.

\section{Sostenibilidad tripartita}

Aludir a la sostenibilidad social se integra a la premisa de la gestión responsable con los grupos de interés, ahora bien, desde la perspectiva de la educación superior esta sostenibilidad se ratifica comola representación misma de la propuesta educativa con la cual desde la educación se proveen cientos de alternativas para participar en la solución conjunta de adversidades por las cuales un grupo social a traviesa, de igual forma, la perspectiva social de la sostenibilidad incrementa la interacción de todos y cada uno de los actores que hacen parte de una comunidad educativa y presenta un llamado a la dirección educativa hacia la articulación de acciones a favor de la corresponsabilidad que implica impactar positivamente la sociedad.

En términos generales, tal como lo indica Litzner-Ordóñez y Rieß (2019), la sostenibilidad social junto con la sostenibilidad económica y ambiental, proveen de un panorama amplio y claramente diversificado a las organizaciones educativas que proponen acciones para contrarrestar las acciones adversas que se generan desde no solo las actividades educativas, sino en general de las acciones del ser humano para con el entorno, para comprender la realidad de algunas universidades latinoamericanas desde esta triple mirada, existen múltiples resultados y comportamientos, donde no hay una tendencia generalizada por favorecer la sostenibilidad, pero si se cuentan con tareas que denotan una mayor sensibilidad y orientación para con definir indicadores, estándares y metas claras en términos de sostenibilidad.

En cientos de centros de educación superior latinos, la sostenibilidad en muy pocas ocasiones se ha volcado para con toda su comunidad educativa, y ejemplo de esto sucede en los programas en los que se pretenden realizar acciones sostenibles con grupos especializados de profesionales y expertos en diferentes disciplinas, lo cual si bien no es equivoco, si identifica un panorama poco articulado que dé cuenta de una verdadera realización de tareas proporcionales a las realidades de cada integrante según Alba-Hidalgo (2017), dicho en otras palabras, en Latinoamérica los pocos resultados en términos de sostenibilidad desde la mirada de las instituciones de educación superior, son el resultado de un pequeño grupo de actores que se dedican exclusivamente a estas tareas, y de esto se rescata que, si bien no es contrario a los resultados representados, si evidencia poco participación colectiva de toda una comunidad educativa, y esta es una de las grandes razones por las cuales la sostenibilidad tiende a ser vista con mayor o menor intensidad en ciertos momentos de la agenda educativa, de allí que sea necesarios mayores esfuerzos a favor de construir una cultura y consciencia social verdadera en cada institución educativa. 
En términos de sostenibilidad, las instituciones educativas de todo tipo sin importar su oferta educativa y filosofía institucional, tienen la posibilidad de favorecer espacios para proponer proyectos y programas de sostenibilidad, que den cuenta de la interacción entre:

- Estudiantes

- Docentes

- Administrativos

- Comunidad externa

- Gobierno y sector público

Ahora bien, el talón de Aquiles que sobre sale en muchos centros de educación, se ve reflejado en la poca vinculación de todas las actividades tanto académicas, como curriculares y administrativas, a favor de una cultura sostenible; de allí que según González-Gaudiano, MeiraCarte, y Martínez-Fernández (2015) el futuro que se presenta para la educación superior latina, identifica una ardua labor para integrar la organización educativa de cara a los desafíos que propone la sostenibilidad, y es así como la postura que deben asumir las universidades no puede ser únicamente desde una mirada local, sino todo lo contrario, deben visualizarse como actores protagónicos de la agenda internacional, ya que la sostenibilidad y el futuro de la humanidad no se puede localizar en una región o lugar del mundo, sino que debe dar cuenta de una coherencia social, económica y ambiental, es por esto que escenarios como la internacionalización de la educación superior posibilitan que las organizaciones educativas amplíen su ámbito de actuación, y a la vez su comunidad educativa se proyecte en un horizonte de tiempo enmarcado en un corto, mediano y largo plazo con objetivos y metas claras.

\section{CONSIDERACIONES FINALES}

Comprender la internacionalización como un elemento clave que contribuye positivamente en la sostenibilidad al interior de las organizaciones educativas, es el llamado que se genera a las universidades latinas, porque desde una mirada holística del contexto latinoamericano se ha identificado que las prácticas de internacionalización no siempre guardan una relación cercana con la sostenibilidad, y más grave aún, la responsabilidad social universitaria RSU en muchos casos es vista como un entorno ajeno a las situaciones que se viven en la vida universitaria en términos de internacionalización, dejando a un lado los grupos de interés que se presentan en un ecosistema educativo internacional, situación que a partir de la pandemia generada por el COVID-19 ha volcado a las instituciones educativas a comprender la necesidad de adoptar mecanismo disruptivos para favorecer el intercambio de experiencias, situación que se ve reflejada en el aumento de clases espejo, congresos online, encuentros con expertos de todo el mundo conectados en la web, entre otros; situaciones que permiten identificar que la codificación tradicional de la educación y la internacionalización tuvieron que cambiar a raíz de la pandemia que tocó a todo el mundo.

La labor de las universidades latinas requiere una reconfiguración de sus labores administrativas y educacionales, esto con la finalidad de redefinir su planeación educativa y fortalecer el vínculo entre los stakeholders y la administración educativa, por lo tanto, no basta con presentar acciones de sostenibilidad y presentar resultados de impacto sostenible, sino que 
se debe presentar una articulación clara para con los sistemas de educación en conjunto, con los actores de cada comunidad educativa; ahora bien, la articulación que se requiere debe darse con suma urgencia, toda vez, que con recurrencia son menos visibles los impactos que se identifican desde la educación superior en Latinoamérica para con la consolidación de la sostenibilidad en la sociedad, ejemplo de esto son los precarios aportes en el cumplimiento de los objetivos de desarrollo sostenible ODS en la agenda 2030.

La internacionalización de la educación superior debe ampliar mucho más su protagonismo en la vida universitaria latina, es por esto que no se debe condicionar la internacionalización educativa únicamente con movilidad académica y la tradicional experiencia de vida internacional, más aún cuando los desafíos del siglo XXI y situaciones como la pandemia generada por el COVID-19 ratifican la necesidad de disminuir la movilidad física entre ciudadanos de diferentes países, y es por esto, que la humanidad y el entorno latino deben hacer uso de los cientos de alternativas tecnológicas e hibridas que proponen las tecnologías de la información y comunicación TIC, en las que no se puede limitar la internacionalización únicamente a una movilidad física, ratificando que el confinamiento del cuerpo no aplica a confirmar la mente.

Un reto que presenta la pandemia del COVID-19 en términos de sostenibilidad, se ha concentrado en la relevancia de la educación superior representa para con el desarrollo de las regiones, de allí que para muchas universidades la pandemia representó un nuevo entorno para posibilitar el impacto a públicos distantes desde las mediaciones tecnológicas, y de igual forma, se configuró como un motor de cambios sociales que proveen de soluciones a los contextos en los que toda una comunidad educativa tiene incidencia, representada por sus estudiantes, administrativos, docentes, entre otros. Lo cierto es que situaciones adversas en las que lo tradicional debe tener un cambio, aun es concebido como una amenaza y en muy pocas ocasiones como un oportunidad, y es así, como la pandemia se configuró como un detonante de la mentalidad descriptiva, invitando a comprender las realidades desde ópticas posiblemente impensadas.

No existe una única ruta para articular las prácticas de internacionalización educativa en las universidades latinas, pero si se pueden generar interacciones claras en el marco de la realidad que se vive por los cientos de cambios sociales, económicos, políticos, tecnológicos, entre otros; es por esto que cada vez más la internacionalización debe desarrollarse desde un camino enriquecido por todo el actuar educativo y no puede ser visto solamente como un complemento más, situación similar para con la sostenibilidad, la cual no puede ser vista como un medio sino como un fin. 


\section{REFERENCIAS BIBLIOGRÁFICAS}

Alba-Hidalgo, D. (2017). Hacia una fundamentación de la sostenibilidad en la educación superior. Revista Iberoamericana De Educación, 73, 15-34. doi:10.35362/rie730197

Arias-Velandia, N. (2018). Aportes a la investigación sobre educación superior virtual desde América Latina: comunicación, redes, aprendizaje y desarrollo institucional y social. Bogotá D.C.: Editorial Politécnico Grancolombiano.

Aznar-Minguet, P., UII, A. M., Piñero, A., y Martínez-Agut, M. P. (2014). La sostenibilidad en la formación universitaria: Desafíos y oportunidades. Educación XX1, 17(1), 131-158. doi:10.5944/educxx1.17.1.10708.

Barba-Álvarez, A. (2012). Instituciones de educación superior, políticas públicas y organización: Editorial Miguel Ángel Porrúa.

Blanco, A., Alba-Ferré, E., y Asensio, E. (2016). Desarrollo y evaluación de competencias en educación superior: Narcea Ediciones.

Brown, S., y Pickford, R. (2016). Evaluación de habilidades y competencias en educación superior: Narcea Ediciones.

Brunner, J. (2010). Educación superior en América Latina: una agenda de problemas, políticas y debates en el umbral del año 2000: CLACSO.

Castro, A. (2018). Reflexiones sobre los estudios generales en la educación superio: Universidad del Norte.

Chacón, R., Montbrun, N., y Rastelli, V. (2009). La educación para la sostenibilidad: Rol de las universidades. Argos, 26(50), 50-74.

Chirinos-Araque, Y., y Pérez-Peralta, C. (2016). La responsabilidad social universitaria: emprendimiento sostenible como impacto de intervención en comunidades vulnerables. Revista Emprendimiento (81), 91-110.

Cuenca, R. (2013). Educación superior, movilidad social e identidad: IEP Ediciones.

Gacel-Ávila, J. (2003). La internacionalización de la educación superior: paradigma para la ciudadanía global: Universidad de Guadalajara.

Gijón-Puerta, J., y Crisol-Moya, E. (2012). La internacionalización de la Educación Superior. El caso del Espacio Europeo de Educación Superior. Revista de Docencia Universitaria, 389-414.

González-Gaudiano, E., Meira-Carte, P., y Martínez-Fernández, C. (2015). Sustentabilidad y Universidad: retos, ritos y posibles rutas. Revista de la Educación Superior, 3(175), 69-93.

Hérvas-Gómez, C., Vásquez-Cano, E., Fernández-Batanero, J., y López-Meneses, E. (2019). Innovación e investigación sobre el aprendizaje ubicuo y móvil en la educación superior. Barcelona: Ediciones Octaedro, S.L. 
Jones, E., y Brown, S. (2016). La internacionalización de la educación superior: perspectivas institucionales, organizativas y éticas: Narcea Ediciones.

Litzner-Ordóñez, I., y Rieß, W. (2019). La Educación para el DesarrolloSostenible en la universidad boliviana. Percepciones del profesorado. Teoría de la Educación, 31(1), 49-173.

López, G. (2014). La internacionalización de la educación superior y la formación de ciudadanos del mundo, ciudadanos glocales. Sophia, 64-69.

López-Segrera, F. (2006). Escenarios mundiales de la educación superior: CLACSO.

Medina, I. L. (2019). Diversidad cultural desde la sociología de la educación, la inmigración en Latinoamérica. Revista Caribeña de Ciencias Sociales, 1-14.

Medina-Alvarado, I. (2019). La formación docente desde los retos de las políticas educativas en la sociedad latinoamericana. Revista Caribeña de Ciencias Sociales, 1-10.

Meneses, T., y Perea, C. (2012). Responsabilidad social universitaria como perspectiva ética en la educación superior latinoamericana. Revista Temas, 141-151. doi:10.15332/rt.v0i6.703

Meza-Aliaga, R. (2020). Los siete aspectos fundamentales para la educación del siglo XXI según Edgar Morin. Una mirada desde la complejidad y la teoría de sistemas. Revista Enfoques Educacionales, 17(1), 91-115.

Monarca, H., y Prieto, M. (2018). Calidad de la educación superior en Iberoamérica. Madrid: Dykinson.

Parrado-Castañeda, Á., y Trujillo-Quintero, H. (2015). Universidad y sostenibilidad: una aproximación teórica para su implementación. AD-minister (26), 149-163.

Pedroza-Flores, R. (2005). Flexibilidad académica y curricular en las instituciones de educación superior: Editorial Miguel Ángel Porrúa.

Ricardo-Barreto, C., y Iriarte-Díazgranados, F. (2017). Las TIC en la educación superior: experiencias de innovación: Universidad del Norte.

Salazar, J. M. (2012). Modelos de aseguramiento de la calidad en la educación superior: RIL editores.

Vallaeys, F. (2018). Las diez falacias de la Responsabilidad Social Universitaria. Revista Digital de Investigación en Docencia Universitaria, 34-58. 